\title{
Dust production and mass loss in cool evolved stars
}

\author{
M.L. Boyer ${ }^{1,2,3, \star}$ \\ 1 Space Telescope Science Institute, 3700 San Martin Drive, Baltimore, MD 21218, USA \\ 2 Observational Cosmology Lab, Code 665, NASA Goddard Space Flight Center, Greenbelt, MD 20771, USA \\ 3 Oak Ridge Associated Universities (ORAU), Oak Ridge, TN 37831, USA
}

Received 2012 Aug 22, accepted 2012 Dec 5

Published online 2013 Feb 1

Key words galaxies: individual (SMC, LMC) - stars: AGB and post-AGB - stars: mass loss

Following the red giant branch phase and the subsequent core He-burning phase, the low- to intermediate-mass stars $\left(0.8<M / M_{\odot}<8\right)$ begin to ascend the asymptotic giant branch (AGB). Pulsations levitate material from the stellar surface and provide density enhancements and shocks, which can encourage dust formation and re-processing. The dust composition depends on the atmospheric chemistry (abundance of carbon relative to oxygen), which is altered by dredging up newly formed carbon to the surface of the star. I will briefly review the current status of models that include AGB mass loss and relate them to recent observations of AGB stars from the Surveying the Agents of Galaxy Evolution (SAGE) Spitzer surveys of the Small and Large Magellanic Clouds, including measures of the total dust input to the interstellar medium from AGB stars.

(c) 2013 WILEY-VCH Verlag GmbH \& Co. KGaA, Weinheim

\section{Introduction}

Stellar mass loss occurs at many places on the HertzprungRussell diagram, but only in cool evolved stars will this mass loss be accompanied by substantial dust production. These stars have long been thought to be among the dominant dust producers in the Universe (e.g., Gerhz 1989), though it has only recently become possible to measure the dust input from entire populations of cool evolved stars into the interstellar media (ISM) of their host galaxies. In this work, I briefly review some recent advances in the models of these stars and their mass loss and summarize recent work that strives to quantify the stellar dust production in the Magellanic Clouds.

\subsection{Stellar mass loss}

Stars with $0.8 \leq M / M_{\odot} \leq 8$ experience this dusty mass loss during the asymptotic giant branch (AGB) phase, after having exhausted core helium, through a pulsationenhanced, dust-driven wind (e.g., Bowen 1988; Winters et al. 2000, 2003; Schirrmacher et al. 2003; Woitke 2006; Mattsson et al. 2008; van Loon et al. 2008). AGB stars experience both thermal pulsations and hydrodynamic pulsations (Vassiliadis \& Wood 1993). Thermal pulses are caused by instabilities in the thin hydrogen- and helium-burning shells around the carbon/oxygen core and result in the thirddredge up (3DUP; Iben 1975; Sugimoto \& Nomoto 1979), during which the star dredges newly-synthesized material from these shells up to the surface of the star. Every dredgeup event causes an increase in the $\mathrm{C} / \mathrm{O}$ ratio at the stellar

\footnotetext{
^ Corresponding author: martha.boyer@nasa.gov
}

surface, affecting the chemistry of the subsequent dust production. The hydrodynamic pulses levitate this material in the stellar atmosphere where it cools to form C- or O-rich molecules and dust grains. Radiation pressure on the dust grains then drives a strong stellar wind, dragging the gas with it through momentum coupling.

It is difficult to model the mass loss process in stellar evolution models, and models have often over or underestimated both the numbers of AGB stars and their brightnesses owing to uncertainties in the onset and strength of mass loss. Recent work by Girardi et al. (2010), however, has shown a significant improvement in the ability of the Padova models to reproduce the stellar populations of galaxies with $[\mathrm{Fe} / \mathrm{H}] \leq 1.2$ and ages less than $3 \mathrm{Gyr}$ by including mass loss that occurs before significant dust production (cf. Schröder \& Cuntz 2005). This results in both a shorter lifetime and a smaller final mass for low-mass stars, and agrees with white dwarf mass measurements made in M 4 by Kalirai et al. (2009).

\subsection{The dust-driven wind}

The efficiency of the 3DUP determines whether an AGB star will become C-rich (e.g., Marigo, Girardi \& Bressan 1999; Zhukovska, Gail \& Trieloff 2008). As carbon is brought to the surface, it ties up free oxygen into $\mathrm{CO}$ molecules; if the ratio of $\mathrm{C}$ to $\mathrm{O}$ surpasses unity, excess carbon is free to form $\mathrm{C}$-rich dust, such as amorphous carbon dust. Metal-poor stars become C-rich more easily than metal-rich stars, since less oxygen is available to tie-up the dredged up carbon. The formation of amorphous carbon dust may not be affected by the initial metal content of the 
star because carbon is formed in-situ, though it is yet unclear what affect the metallicity has on carbon dust formation (e.g., van Loon et al. 2008; Sloan et al. 2008). The formation of other types of $\mathrm{C}$-rich dust, such as $\mathrm{SiC}$, is limited by the availability of heavier metals and thus by the metallicity of the star.

AGB stars with an inefficient 3DUP remain O-rich, and the more metal-rich of these stars can form silicate dust. Red supergiant stars with $8 \leq M / M_{\odot} \leq 25$ do not dredge up carbon, so remain O-rich and can form dust that is very similar to O-rich AGB stars (e.g., Kastner et al. 2008; Woods et al. 2011).

Amorphous carbon dust is optically thick near $1 \mu \mathrm{m}$ and it forms close to the star $\left(2-3 R_{*}\right)$, so it can easily drive a strong stellar wind. Forsterite $\left(\mathrm{Mg}_{2} \mathrm{SiO}_{4}\right)$ dust also forms close to the star, but is too optically thin to drive a wind in $\mathrm{O}$ rich stars. Höfner (2008) propose that O-rich stellar winds could be driven by photon scattering by iron-free silicates if the grains are large $(\sim 1 \mu \mathrm{m})$, though that work questioned whether enough material is available to form large grains at these radii. Recent spatially-resolved, multi-wavelength observations of scattered light from circumstellar dust shells of 3 nearby O-rich AGB stars reveal dust shells containing $0.6 \mu \mathrm{m}$ grains at $<2 R_{*}$ (Norris et al. 2012). If confirmed, these observations suggest that large iron-free grains may indeed be driving O-rich winds via photon scattering.

\section{AGB stars and dust production in the Magellanic Clouds}

\subsection{Description of the data and stellar classification}

Photometry of point-sources in the Magellanic Clouds was compiled by the Spitzer Space Telescope Surveying the Agents of Galaxy Evolution (SAGE) Legacy team (Meixner et al. 2006; Gordon et al. 2011). Optical data is from the Magellanic Clouds Photometric Survey (MCPS; Zaritsky et al. 2007) and near-infrared (IR) data is from the two-micron all-sky survey (2MASS; Skrutskie et al. 2003). Photometry at 3.6, 4.5, 5.8, and $8 \mu \mathrm{m}$ was obtained with the InfraRed Array Camera (IRAC; Fazio et al. 2004) and photometry at 24 and $70 \mu \mathrm{m}$ was obtained with the Multi-band Imaging Photometer for Spitzer (MIPS; Rieke et al. 2004). The SMC spatial coverage $\left(\approx 30 \mathrm{deg}^{2}\right)$ includes the full bar, wing, and tail. The spatial coverage of the LMC includes the central $\approx 50 \mathrm{deg}^{2}$.

To select each type of star, we use the color cuts described by Boyer et al. (2011). Less dusty stars are selected via their near-IR colors (also see Cioni et al. 2006) and separated into red supergiants (RSGs), carbon-rich AGB (C-AGB) and oxygen-rich AGB (O-AGB) stars. Dusty "extreme" AGB stars (x-AGB stars) that are undetected or misidentified in the near-IR (cf. Boyer et al. 2009) are recovered via their [3.6] - [8] color.

Far-infrared (FIR) objects are those whose $24 \mu \mathrm{m}$ fluxes exceed their $8 \mu \mathrm{m}$ fluxes. Some of these may be very dusty
Table 1 The Number of AGB and RSG Stars in the Magallanic Clouds from Boyer et al. (2011).

\begin{tabular}{lrr}
\hline Stellar Type & $N_{\text {LMC }}$ & $N_{\text {SMC }}$ \\
\hline C-AGB & 6212 & 1729 \\
O-AGB & 17242 & 3722 \\
X-AGB & 1105 & 349 \\
RSG & 4604 & 3325 \\
FIR & 224 & 57 \\
\hline
\end{tabular}

AGB stars, but most are likely background AGN or young stellar objects (Boyer et al. 2011). We include them here as a separate category of sources. Table 1 lists the numbers of each source type (Boyer et al. 2011).

\subsection{AGB contribution to the global flux}

Since we have compiled a complete (both in sensitivity and in spatial coverage) sample of AGB stars in the Magellanic Clouds, we can compare the total AGB flux to the global flux of each galaxy. The results are shown in Fig. 1 (Melbourne \& Boyer 2013). We find that the AGB star contribution peaks at $\approx 20 \%$ near $3-5 \mu \mathrm{m}$ in both galaxies. The Orich star contribution peaks at bluer wavelengths $(\approx 2 \mu \mathrm{m})$ and $\mathrm{C}$-rich stars contribute more at redder wavelengths. The contribution of the $\mathrm{x}$-AGB stars, which are mostly C-rich, peaks at $8 \mu \mathrm{m}$ in the $\mathrm{SMC}$ at $\sim 8 \%$, despite their very small numbers (Table 1). In the LMC, the $\mathrm{x}$-AGB contribution at $8 \mu \mathrm{m}$ is $<2 \%$, presumably due to the strong presence of polycyclic aromatic hydrocarbons (PAHs) in the ISM, which dominate the $8 \mu \mathrm{m}$ flux (Bot et al. 2010).

These results suggest that the $1-\mu \mathrm{m}$ region may be the most useful for measuring the mass-to-light ratios of galaxies, since the AGB and RSG contribution is low at these wavelengths and since this region of the spectrum is not strongly impacted by extinction from dust. Star formation histories derived from the rest-frame $8 \mu \mathrm{m}$ flux may also be strongly affected by the presence of AGB stars.

\subsection{Stellar dust production in the Magellanic Clouds}

To estimate the dust mass-loss rates (or the dust-production rates) of AGB and RSG stars, we use the $8-\mu \mathrm{m}$ excess $\left(X_{8} \mu \mathrm{m}\right)$, which was shown to be a good proxy for the dust mass-loss rate by Srinivasan et al. (2009). To compute $X_{8} \mu \mathrm{m}$, we fit each star's optical and near-IR spectral energy distribution to either an O-rich or C-rich model photosphere (Gautschy-Loidl et al. 2004; Hauschildt et al. 1999) and measured the $8-\mu \mathrm{m}$ flux above the photosphere. We selected a representative sample of the stars that have additional data (either AKARI mid-IR photometry or IR spectroscopy from Spitzer) for detailed radiative transfer modeling in order to calibrate the relationship between the dust-production rate and the $8-\mu \mathrm{m}$ excess and extrapolated this relationship to the entire AGB and RSG population. See Boyer et al. (2012) for more details. 


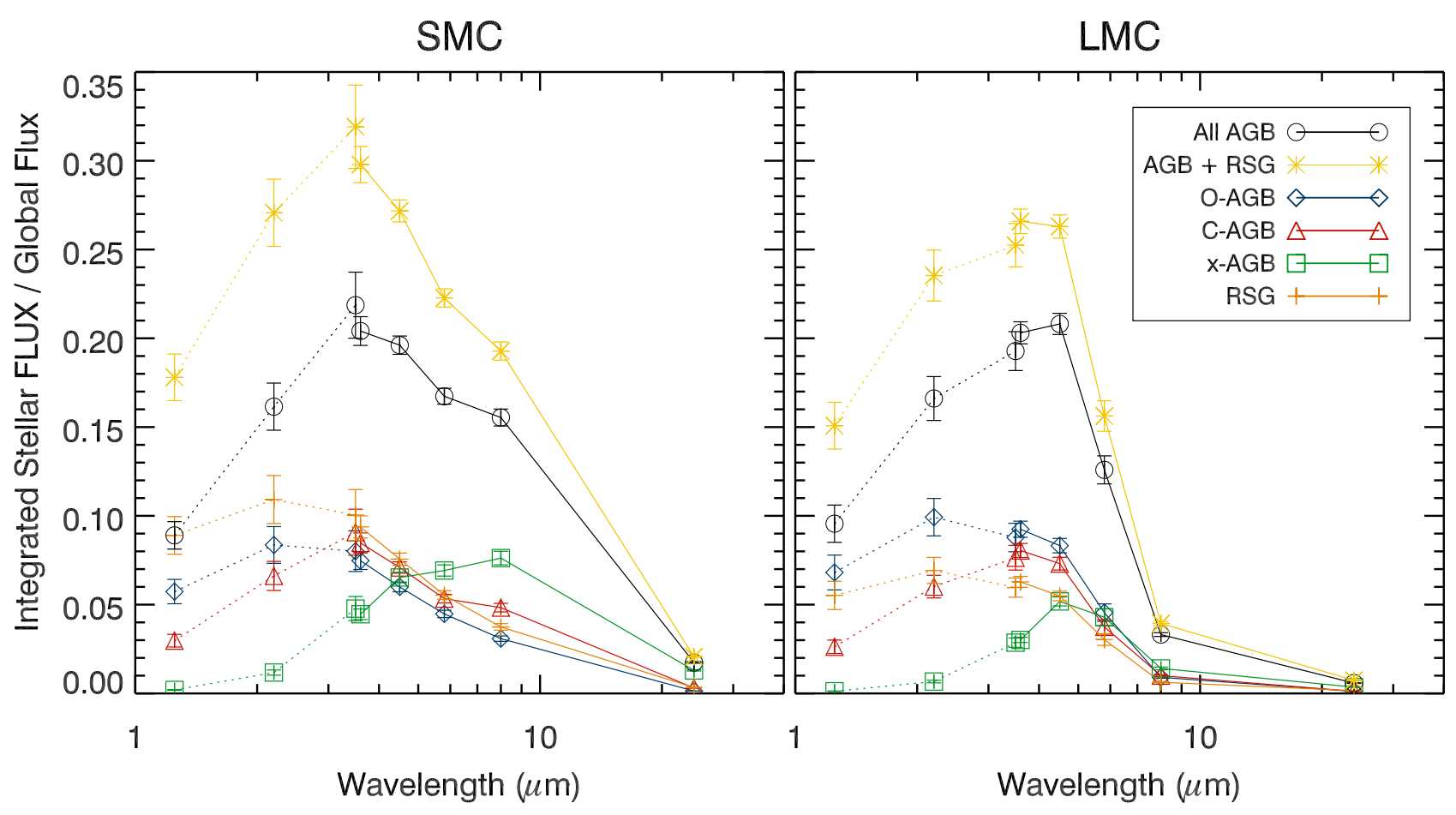

Fig. 1 (online colour at: www.an-journal.org) The contribution of AGB and RSG stars to the global flux of the Magellanic Clouds as a function of wavelength, adapted from Melbourne \& Boyer (2013). The global fluxes are from Isreal et al. (2010) and Gordon et al. (2011); 1-3.5 $\mu \mathrm{m}$ global fluxes were measured using COBE images (dotted lines) and the 3.6-24 $\mu \mathrm{m}$ fluxes are from Spitzer images (solid lines). The AGB contribution peaks near 3-5 $\mu \mathrm{m}$ with C-rich stars contributing more at redder wavelengths. The $\mathrm{x}$-AGB stars contribute less at $8 \mu \mathrm{m}$ in the LMC owing to a strong PAH feature captured in the $8 \mu \mathrm{m}$ band.

More recent work in the LMC (Riebel et al. 2012) and SMC (Srinivasan et al. 2013, in preparation) use the newly developed Grid of AGB and RSG Stars (GRAMS; Sargent et al. 2011; Srinivasan et al. 2011) to estimate the dustproduction rates in both galaxies. This technique takes into account the whole SED of each individual star and is thus more robust. The dust-production rates from the GRAMS models are 2-4 times larger with those found using the $8-\mu \mathrm{m}$ excess. For both techniques, several factors contribute to the uncertainties in the derived dust-production rates, including the uncertainties in stellar properties such as variability and dust composition and in the adopted model parameters such as optical constants, dust shell geometry, and outflow velocity. See Boyer et al. (2012) for a detailed discussion.

Figure 2 shows the cumulative dust-production rate of AGB and RSG stars in the LMC and SMC from Boyer et al. (2012). The x-AGB stars contribute nearly an order of magnitude more dust than all other AGB and RSG stars combined, despite comprising only a few percent of the AGB population (Table 1). RSGs contribute the least amount of dust and regular O-AGB and C-AGB stars contribute similar amounts in each galaxy. If FIR sources are all dusty AGB stars, their dust contribution is also substantial, though still less than the $\mathrm{x}$-AGB stars.

\section{Discussion}

Do AGB stars dominate the dust production in the Magellanic Clouds? The dust-production rates presented in Sect. 2.3 only provide the current snapshot of stellar dust production in each galaxy. However, the star formation histories of both galaxies (Harris \& Zaritsky 2004; Rubele et al. 2012) indicate that the current star formation rate has remained constant within a factor of 3 over the expected dust lifetime, so it is reasonable to assume that the AGB dust injection rate to the ISM has remained roughly constant.

If supernovae ( $\mathrm{SNe}$ ) are the drivers of dust destruction, then the SN rate $\left(\tau_{\mathrm{SN}}\right)$ can be used to estimate the dust lifetime in the ISM. Following Draine (2009), we assume that a typical SN with energy $E_{0}=10^{51} \mathrm{erg}$ in a medium with density $n_{\mathrm{H}}=1 \mathrm{~cm}^{-3}$ will remain in the Sedov-Taylor phase until the shock speed drops to $200 \mathrm{~km} \mathrm{~s}^{-1}$. Each SN therefore processes $M \approx E_{0} / v_{s}^{2}=1260 \mathrm{M}_{\odot}$ of interstellar material. We thus expect that the dust lifetime within the ISM is

$\tau_{\mathrm{d}}=\frac{M_{\mathrm{ISM}}^{\mathrm{gas}}}{\left(1260 M_{\odot} / \tau_{\mathrm{SN}}\right)}$

where $M_{\mathrm{ISM}}^{\mathrm{gas}}\left(\mathrm{H} 1+\mathrm{H}_{2}\right)$ is $4.5 \times 10^{8} \mathrm{M}_{\odot}$ (Bolatto et al. 2011). This results in a dust lifetime of $(3.0-8.6) \times 10^{8} \mathrm{yr}$ (see Boyer et al. 2012 for more details). 

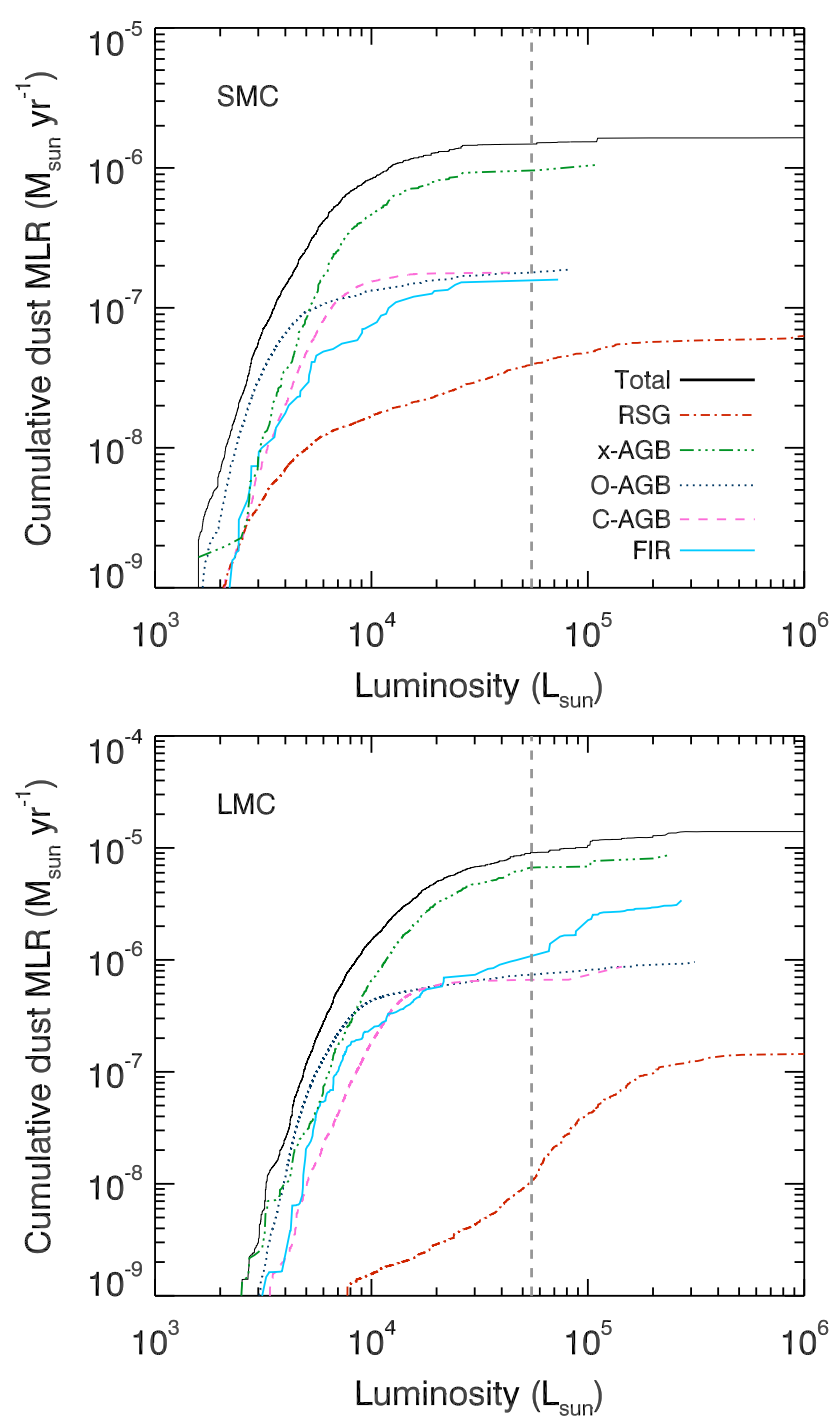

Fig. 2 (online colour at: www.an-journal.org) Cumulative dust mass-loss rates (MLRs) for AGB and RSG stars in the Magellanic Clouds, adapted from Boyer et al. (2012). The x-AGB stars contribute significantly more dust than the other stars in both galaxies, despite comprising only a few percent of the dust-producing population (Table 1). The nature of the far infrared (FIR) sources is unknown, but they may be YSOs. They are included here as if they were C-rich AGB stars. The vertical dashed line marks the classical AGB limit.

A comparison of the dust lifetime combined with the stellar dust input to the observed ISM dust mass (0.291.1) $\times 10^{-6} \mathrm{M}_{\odot}$; Bot et al. 2010) shows that the AGB stars combined can account for only $\approx 2 \%$ of the ISM dust in the SMC and $3 \%$ in the LMC (see Matsuura et al. 2009 for a detailed discussion of the LMC). Even if no dust is destroyed in the ISM, the AGB stars can account for $<5 \%$ of the ISM dust. If using the GRAMS-derived dust-production rates, these percentages could be 2-4 times larger.

It is possible that by considering only wavelengths $<24 \mu \mathrm{m}$, we are underestimating the dust production around AGB stars by neglecting colder dust. Preliminary searches for cold dust shells around AGB and RSG stars in the LMC using the Herschel Space Telescope have resulted in the detection of only one cool evolved star at $\lambda>70 \mu \mathrm{m}$ : the RSG IRAS 05280-6910. However, while detected, this star shows now additional far-IR excess, indicating that all dust was accounted for at Spitzer wavelengths (Boyer et al. 2010). Very cold dust may thus be rare around AGB and RSG stars and is not a likely solution.

These results indicate that dusty evolved stars cannot be the sole dust contributors to the ISM. There are at least two other possible dust sources: SNe and ISM grain growth. The dust production by $\mathrm{SNe}$ is highly uncertain, though to account for the missing dust, $\mathrm{SNe}$ would have to be highly efficient dust producers. Only one SN (SN 1987A) has shown evidence for the required significant dust production $\left(\approx 0.7 \mathrm{M}_{\odot}\right.$; Matsuura et al. 2011), and those results are still unconfirmed. Dust accretion in molecular clouds (e.g., Dwek 1998; Zhukovska, Gail \& Trieloff 2008) from seeds provided by stellar sources is a plausible solution to this missing dust problem.

While studying AGB stars in the Magellanic Clouds provides valuable insight into stellar dust production, these galaxies represent a limited range in metallicity, star formation history, interaction history, and age. For a full understanding of how these parameters affect AGB dust production, these studies must be expanded to other resolvable galaxies. Ongoing studies using Spitzer and Hubble Space Observatory observations of Local Group dwarf galaxies (DUsty STars In Nearby Galaxies with Spitzer - or DUSTINGS; Boyer et al. 2013, in preparation) and of M31 (The Panchromatic Hubble Andromeda Treasury program - or PHAT; PI: J. Dalcanton) are currently underway.

\section{Concluding remarks}

AGB stars are the most efficient known dust producers. We estimate the dust injection rate from the complete populations of AGB and RSG stars in the LMC and SMC and compare this to the current ISM dust mass. We find that C-rich AGB candidates account for about $80-90 \%$ of the total cool evolved star dust input in both galaxies, especially from the "extreme" C-rich AGB stars.

Despite these high dust-production rates, AGB and RSG dust can account for only a few percent of the ISM dust, suggesting that $\mathrm{SNe}$ must create significant amounts of dust (such as what is inferred from far-IR observations of SN 1987A) or dust must grow in dense molecular clouds in the ISM itself.

Acknowledgements. This work is supported by NASA via JPL contracts 130827 and 1340964. Many thanks to the CoolStars17 conference organizers and attendees for an excellent conference. 


\section{References}

Bolatto, A. B., Leroy, A. K., Jameson, K., et al. 2011, ApJ, 741, 12

Bot, C., Ysard, N., Paradis, D., et al. 2010, A\&A, 523, 20

Bowen, G. H. 1988, ApJ, 329, 299

Boyer, M. L., Skillman, E. D., van Loon, J. Th., et al. 2009, ApJ, 697, 1993

Boyer, M. L., Sargent, B. A., van Loon, J. Th., et al. 2010, A\&A, 518,142

Boyer, M. L., Srinivasan, S., van Loon, J. Th., et al. 2011, AJ, 142, 103

Boyer, M. L., Srinivasan, S., Riebel, D., et al. 2012, ApJ, 748, 40

Cioni, M.-R. L., Girardi, L., Marigo, P., \& Habing, H. J. 2006, A\&A, 448, 77

Dwek, E. 1998, ApJ, 501, 643

Fazio, G. G., Hora, J. L., Allen, L. E., et al. 2004, ApJS, 154, 10

Gautschy-Loidl, R., et al. 2004, A\&A, 422, 289

Gehrz, R. D. 1989, Sources of Stardust in the Galaxy, in Interstellar Dust, ed. L. J. Allamandola, \& A. G. G. M. Tielens, IAU Symp. 135 (Cambridge Univ. Press), 445

Girardi, L., Williams, B. F., Gilbert, K. M., et al. 2010, ApJ, 724, 1030

Gordon, K. D., Meixner, M., Meade, M. R., et al. 2011, AJ, 142, 102

Harris, J., \& Zaritsky, D. 2004, AJ, 127, 153

Hauschildt, P. H., Allard, F., \& Baron, E. 1999, ApJ, 512, 377

Höfner, S. 2008, A\&A, 491, 1

Iben, I. 1975, ApJ, 196, 525

Isreal, F., P., Wall, W. F., Raban, D., et al. 2010, A\&A, 519, A67

Kalirai, J. S., Saul Davis, D., Richer, H. B., et al. 2009, ApJ, 705, 408

Kastner, J. H., Buchanan, C., Sahai, R., et al. 2008, AJ, 136, 1221

Marigo, P., Girardi, L., \& Bressan, A. 1999, A\&A, 344, 123

Matsuura, M., Barlow, M. J., Zijlstra, A. A., et al. 2009, MNRAS, 396,918
Matsuura, M., Dwek, E., Meixner, M., et al. 2011, Sci, 333, 1258

Mattsson, L., Wahlin, R., Höfner, S., \& Eriksson, K. 2008, A\&A, 484,5

Meixner, M., Gordon, K. D., Indebetouw, R., et al. 2006, AJ, 132, 2268

Melbourne, J., \& Boyer, M. L. 2013, ApJ, in press

Norris, B. R. M., Tuthill, P. G., Ireland, M. J., et al. 2012, Nature, 484, 7393

Riebel, D., Srinivasan, S., Sargent, B., \& Meixner, M. 2012, ApJ, 753,71

Rieke, G. H., Young, E. T., Engelbracht, C. W., et al. 2004, ApJS, 154,25

Rubele, S., Kerber, L., Girardi, L., et al. 2012, A\&A, 537, A106

Sargent, B. A., Srinivasan, S., \& Meixner, M. 2011, ApJ, 728, 93

Schirrmacher, V., Woitke, P., \& Sedlmayr, E. 2003, A\&A, 404, 267

Schröder, K.-P., \& Cuntz, M. 2005, ApJ, 630, 73

Sloan, G. C., Kraemer, K. E., Wood, P. R., et al. 2008, ApJ, 686, 1056

Srinivasan, S., Meixner, M., Leitherer, C., et al. 2009, AJ, 137, 4810

Srinivasan, S., Sargent, B. A., \& Meixner, M. 2011, A\&A, 532, 54

Sugimoto, D., \& Nomoto, K. 1979, PASJ, 27, 197

van Loon, J. Th., Cohen, M., Oliveira, J. M., et al. 2008, A\&A, 487, 1055

Vassiliadis, E., \& Wood, P. R. 1993, ApJ, 413, 641

Winters, J. M., Le Bertre, T., Jeong, K. S., et al. 2000, A\&A, 361, 641

Winters, J. M., Le Bertre, T., Jeong, K. S., et al. 2003, A\&A, 409, 715

Woitke, P. 2006, A\&A, 460, 9

Woods, P. M., Oliveira, J. M., Kemper, F., et al. 2011, MNRAS, 411, 1597

Zhukovska, S., Gail, H.-P., \& Trieloff, M. 2008, A\&A, 479, 453 